\title{
COMPARING THE EFFECT OF OCCLUSAL ADJUSTMENT USING T SCAN AND ARTICULATING PAPER ON THE VERTICAL BONE HEIGHT CHANGES AROUND DENTAL IMPLANTS SUPPORTING LOWER SINGLE FIXED DETACHABLE HYBRID PROSTHESIS
}

\author{
Ahmed M. Osama A. Shawky*
}

\begin{abstract}
The Purpose of this study is to is to compare the effect of occlusal adjustment using T scan III and articulating paper on the vertical bone height changes around dental implants supporting lower single fixed detachable hybrid prosthesis
\end{abstract}

Material and method: Fourteen male patients having completely edentulous mandibular arch and full dentate in the maxillary arch were selected from the outpatient clinic prosthodontics department faculty of dentistry Ain Shams University to participate in this study . All Patients were rehabilitated with six implants retaining mandibular fixed detachable prosthesis designed by CAD/ CAM. According to the method of detection of occlusal errors and adjustment the patients were divided into two groups, Group I in which T scan III device was used to detect occlusal errors and group II where articulating paper was used in the patient mouth and occlusal adjustment were made according to the tool used. Radiographic evaluation using CBCT was done to evaluate the amount of vertical bone loss around the supporting implant throughout the follow -up period.

Results : There was increase in vertical bone loss in group I and II through-out the follow up period and on comparing the two groups there was a significant difference in the vertical bone height changes around the supporting implants after one year from the follow up period where group II showed more vertical bone loss.

Conclusion : Within the limitation of this study, it could be concluded that the use of $\mathrm{t}$ scan III device allow for better occlusal adjustment in case of implant supported lower single denture than articulating paper and resulting in less vertical bone loss around the supporting dental implants.

\footnotetext{
* Associate Professor of Removable Prosthodontics, Faculty of Dentistry, Ain-Shams University
} 


\section{INTRODUCTION}

Placement of dental implants in the single edentulous mandible has become a standard treatment for patients who are not satisfied with their conventional complete dentures. ${ }^{(1)}$

A major point affecting the success of a dental implant is the way in which mechanical forces are transferred from the implant to the bone. It is essential that neither the implant nor the supporting bone be stressed beyond the long-term fatigue level. (2)

The role of occlusion and occlusal errors in periimplant bone loss has been suggested that it may cause peri-implant disease and therefore should be avoided ${ }^{(3)}$. Although there are many different opinions of how occlusal overload have negative effect on both the dental implant and the surrounding bone, it also can have mechanical influences on the dental implant and the restoration such as screw loosening, screw fracture or implant texture fracture ${ }^{(4)}$

There are many clinical situations that may cause occlusal overload as in case of premature contacts, occlusal disharmony, long cantilever, parafunctional habits and wrong occlusal scheme. ${ }^{(5)}$

Correct occlusal diagnosis and analysis can be achieved by an objective measurement of occlusion $^{(6)}$. Articulating papers are the most commonly used indicators to locate the occlusal contacts. They differ in terms of width, thickness and the type of the dye impregnated. They are hydrophobic in nature. Articulating papers, despite being the most frequently used occlusion indicators, have the following inadequacies. They are easily ruined by saliva and hence require usage in a dry field. ${ }^{(7)}$

Different methods are available currently for occlusion evaluations; with $\mathrm{T}$ scan being one of the modern technologies to digitally evaluate and diagnose occlusion ${ }^{(8)}$.
The T-Scan occlusal analysis system (Tekscan) is a Microsoft compliant system that can record a given contact sequence in 0.01-s increments. It consists of a piezoelectric foil sensor, a sensor handle, both hardware and software for recording, analyzing and viewing the data. The T-Scan identifies the time magnitude and the distribution of the occlusal contacts. ${ }^{(9)}$

This device is used in many clinical situations when occlusal adjustment is necessary as Complete dentures, fixed or removable partial dentures, Complete arch reconstruction by using implants, Complete arch reconstruction involving FPD, Natural tooth occlusal equilibration, Occlusal splints, Mandibular repositioning devices. ${ }^{(10)}$

Sensitivity, reliability, reproducibility are the major advantages when $\mathrm{T}$ scan is used for the digital occlusal analysis ${ }^{(11,12)}$ as it has the ability to record the very first contact of tooth, relative force and timing ${ }^{(13)}$ The current version of $\mathrm{T}$ scan used recently is T scan III.

The objective of the current study is to is to compare the effect of occlusal adjustment using $\mathrm{T}$ scan III and articulating paper on the vertical bone height changes around dental implants supporting lower single fixed detachable hybrid prosthesis.

\section{MATERIALS AND METHODS}

Fourteen patients having completely edentulous mandibular arches and full dentate maxillary arch with age ranging between 50-65years were selected from the out-patient clinic, Removable Prosthodontics Department faculty of Dentistry Ain Sham University to participate in this study.

The selected patients were free from any systemic diseases that would affect bonemetabolism, had no history of recent administration of chemotherapy or radiotherapy and no history of para-functional habits. 
Visual and digital examinations were thoroughly carried out to assess the condition of intra-oral tissues, the mucosa overlying the edentulous ridge was examined, the opposing teeth were examined for carious lesions, gingival inflammation or pockets were detected and treated.

All patients were evaluated by cone beam radiographs to examine the condition of lower ridge. Evaluation was done for the bone quality and quantity to ensure width with minimum $6 \mathrm{~mm}$ and length with $14 \mathrm{~mm}$ and relation to vital structures as mandibular canal and to ensure the absence of any pathologic lesion or remaining roots.

Preliminary impressions for both arches were made using irreversible hydrocolloid impression material in properly adjusted stock trays and poured into dental stone to produce study casts. A tentative centric jaw relation record was made and upper and lower diagnostic casts were mounted on a mean value articulator to evaluate the available restorative inter arch space to ensure the presence of at least $12 \mathrm{~mm}$ and determining the presence of any over-erupted or tilted teeth, and the required occlusal adjustments.

Opposing upper natural teeth interfering with artificial teeth were adjusted on the cast and accordingly modified in the patient's mouth. Lower primary impressions were made and poured in dental stone to obtain the study casts. Self-cure acrylic resin special trays were constructed on the diagnostic casts.

Border tracing was done using green stick compound $^{*}$ and the secondary impressions were made using zinc oxide/Eugenol impression** material then poured in dental stone to obtain the master cast.
Lower occlusion block was constructed. Facebow record was made to mount the upper cast on semi-adjustable articulator ${ }^{* * *}$ whereas centric occluding relation was used to mount the mandibular cast. Protrusive record was made to adjust the horizontal condylar guidance of the articulator.

Modified cross-linked acrylic teeth having appropriate shade and size were arranged following the mutually protected occlusion with anterior guidance. Blue inlay wax was added to the incisal cusp tip edge of the lower canines, the articulator was moved laterally to curve the wax to create free gliding movements between the upper and lower canines on the working side and disocclusion between all the other teeth. The wax was then replaced by composite by the help of a labial silicon index.

The trial denture was tried in the patient's mouth to ensure proper extension and even contact between all the posterior teeth and harmony between centric occlusion and centric relation at the predetermined vertical dimension of occlusion. The waxed up lower denture was flasked and processed into heat-cured acrylic resin. Laboratory remount was performed to correct any occlusal errors. Denture was delivered to the patient.

Virtual planning began with dual scan protocol by modifying patient's lower complete single denture into radiographic guide by adding several spherical radiographic composite markers in a staggered pattern at different levels to occlusal plane on the buccal and labial flanges of the denture. The first scan was carried out for the lower denture on the cast and the second scan was done while the patient wearing the lower denture and biting in centric occlusion.

\footnotetext{
* Perfectin, S.A.I.C., HUBAC, BUENESAIRES, Argentina

${ }^{* *}$ Cavex impression paste, Cavex Hollad by P.O. Box 852, 2003 RW

${ }^{* * *}$ Bioart, Sao Carlos, Brasil
} 
Based on the spherical radiographic markers visible in both scans, the two scans were superimposed onto each other and the CBCT raw data was converted into 3D information where the final file contained reformatted images in 3D bone model, $3 \mathrm{D}$ radiological dataset and $3 \mathrm{D}$ radiographic modified denture guide model. The Software ${ }^{4 *}$ allows the selection of the implants' location, length and diameter according to the patient's bone quantity and approximation to inferior alveolar nerve. Fig. (1)

Stereolithographic apparatus Envision tec DDDP was used corresponding to slice intervals, a LASER that is mounted on top of vat moves in sequential cross sectional of $1 \mathrm{~mm}$ increments, to produce template.Polymerization process of photo polymerized resin occured in layers.

The 3D surgical guide was provided with metallic sleeves corresponding to the virtually planned drilling sites and contains three labially for fixation screws with an adequate distant form the planned implants' drilling sites so that all patients were rehabilitated by fixed detachable lower single denture retained by six dental implants ${ }^{* *}$.

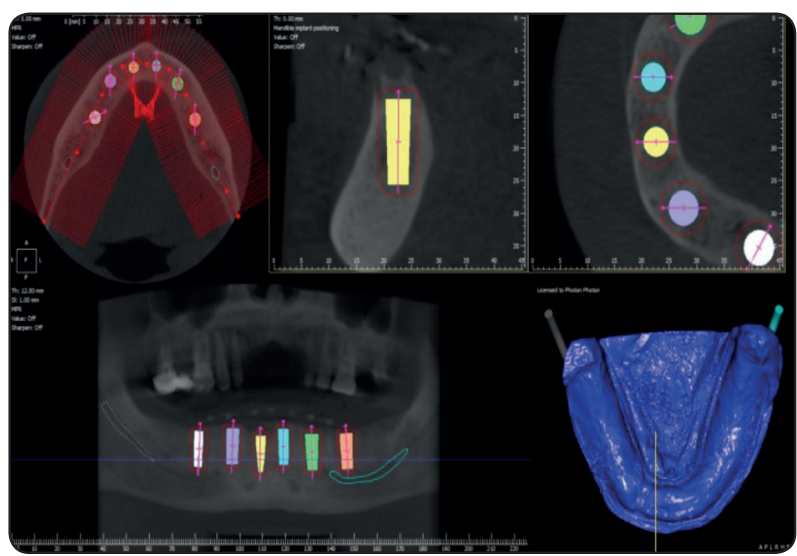

Fig. (1) 3D images showing the selection of the implants' location, length and diameter according to the patient's bone

\footnotetext{
* 2 guide cyber med software, Seoul,Korea

** Interactive Implant, Implant Direct, USA

*** In2 guide universial kit cyber med,Seoul,Korea
}

Surgery was performed under nerve block local block anesthesia Fixation of the mucosa supported guide was done by using the manufacture provided surgical guide kit ${ }^{* * *}$ in2 universal kit cyber med to drill through 3 labial windows to place anchorage fixation pins. Fig. (2)

The drilling sequence with a tissue punch through each metal sleeve of the surgical guide, Following the report provided with the surgical guide about the exact drill length and diameter to be used according to the virtual implant plan. The sequential drilling was done for each implant through the surgical guide finally the implants were inserted through the surgical guide to ensure accurate positioning in the bone as planned in the softwareand the surgical guide was removed from the mouth then gingival formers were screwed to the implants. Fig. (3)

Closed tray impression technique was performed by attaching closed-tray impression copings to implants with an elastomeric impression material. After the impression material was set, the impression was removed, and impression copings were unscrewed from the implants. These unscrewed impression copings were then screwed to implant analogues and together reinserted into the impression.

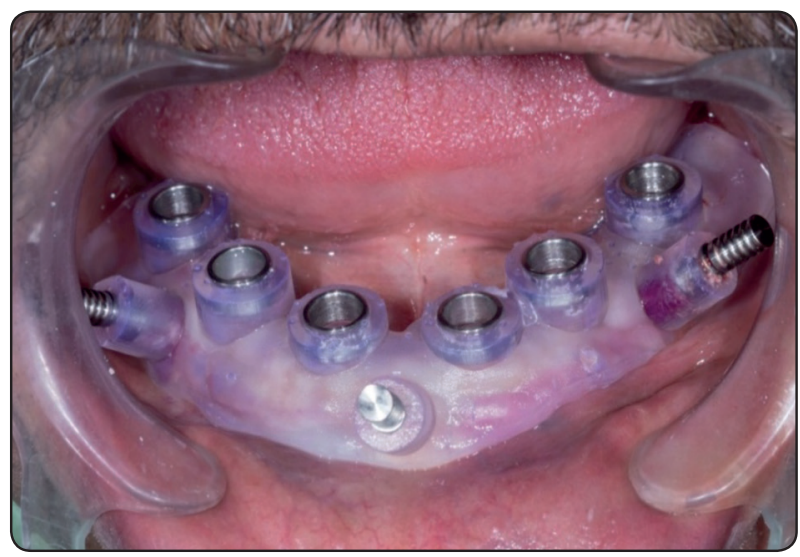

Fig. (2) Fixation of the mucosa supported surgical guide 


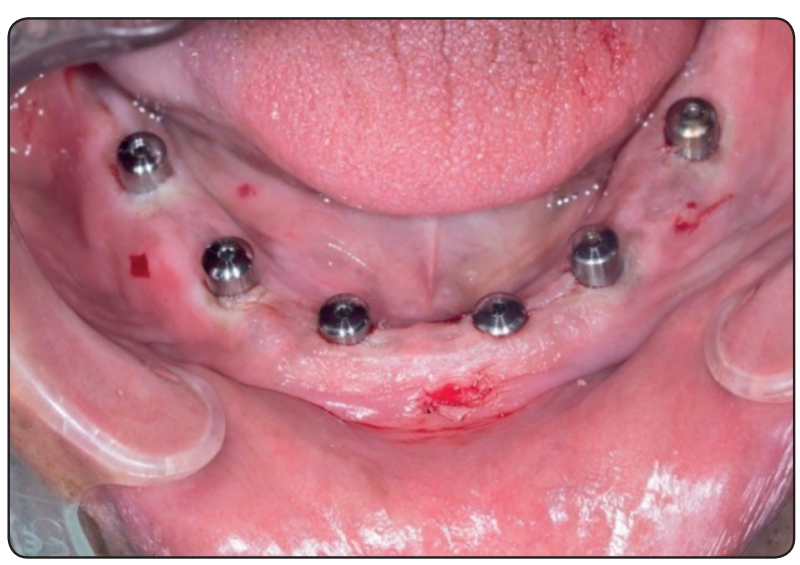

Fig. (3) six implants were inserted in the bone as planned in the software.

Scan abutments were then screwed onto the implant analogues on the stone cast. Desktop extraoral scanner was then used to scan the transfer abutments for detection in the computer software to accurately detect the implant position and depth. The acquired images were then superimposed on the previous scans of the mandibular single denture. Within the computer software, the transfer abutments were then replaced by Titanium bases stored in the library supplied with the software upon which the final prosthesis will be constructed. The prosthesis conforming to the lower single denture was planned and designed.

After the plan was completed, prepolymerized PMMA prosthesis was milled by CAM to be used to check extensions, pressure areas and passive fit was checked visually by using a probe and by taking peri-apical radiographs to check for misfits.

After necessary adjustments were done, the PMMA prosthesis was rescanned then delivered to the patient within 3 days for immediate use throughout the three-month healing period. After healing, The PMMA scan after adjustments was used by the lab for Monolithic Zirconium milling and subsequent veneering. Fig. (4)

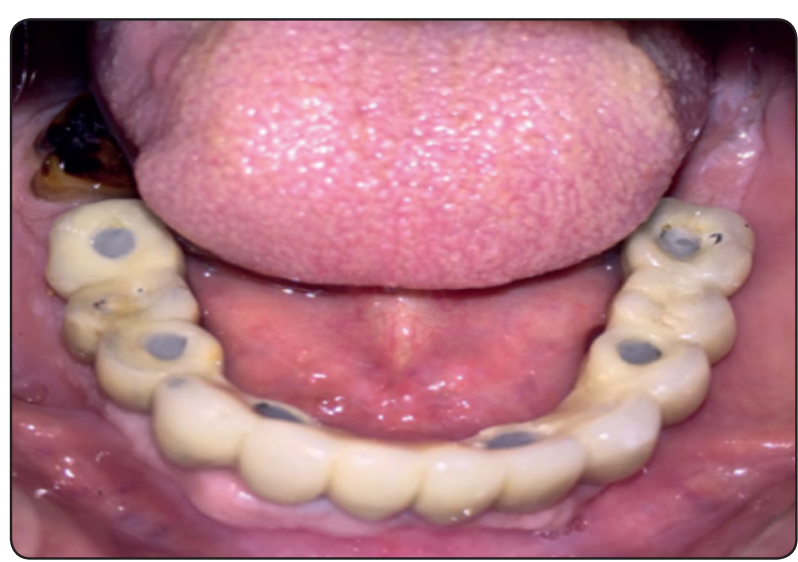

Fig. (4) lower single fixed detachable hybrid prosthesis delivered

After prosthesis delivery, according to the method of detection of occlusal errors and adjustment the patients were divided into two groups.

Group I in which T scan* device was used to detect occlusal errors and adjustment and group II where articulationg paper** was used in the patient mouth.

In group II straight articulating paper was used of thickness $50 \mu$ and width $20 \mathrm{~mm}$ where articulating paper marks were corrected to adjust high spots in centric and eccentric both lateral and protrusive .

In Group I, The proper T-Scan Sensor support and proper size of pressure sensors were chosen and guided by the T-Scan support into the hand piece.

The T-Scan hand piece was then connected to the computer via USB cable, the main window of the software was opened and new patient data were inserted. New movie button was then clicked in the patient record. A blank real time window appeared for the new patient. The sensor was placed in the patient's mouth, with the sensorsupport pointer between the two central incisors and thehandle was kept as parallel to the occlusal plane as possible.

The patient was asked to close and the tooth contact was observed on the screen. Record Start $\&$ Stop button on the handle was pressed to record

\footnotetext{
* Tekscan Inc., South Boston, MA USA

** Articulating paper Products Dentaires SA Vevey, Switzerland
} 
and the status bar was then displayed "Rec. The patient was instructed to bite down normally on thesensor for 2 seconds and then opened slowly. Three recordswill be taken for each patient.

As soon as recording was completed, the Realtime windowbecame a 2-D Movie window, which was divided into two equal colored boxes (one green for the left side and one red forthe right side) around the mid-sagittal plane showing the difference between the intensity of the biting forces on the both sides.

A 3-D Movie window, Graph window and Graph Zoom window were also automatically opened for the current movie.The Graph and Graph Zoom windows contain color-coded "traces" representing the forces applied on each teeth,the magnitude of forces applied, the distribution of the forces along the arch and teeth under heavy contact and premature contact inside each of the coloredboxes in the 2-D Movie window. Fig. (5)

According to the data displayed on the 3D graph, teeth with premature contact were reduced and the process was repeated until nearly the contact between posterior teeth is equal in distribution and intensity and until the intensity of the biting force of both sides of the arch are nearly equal. Fig. (6)

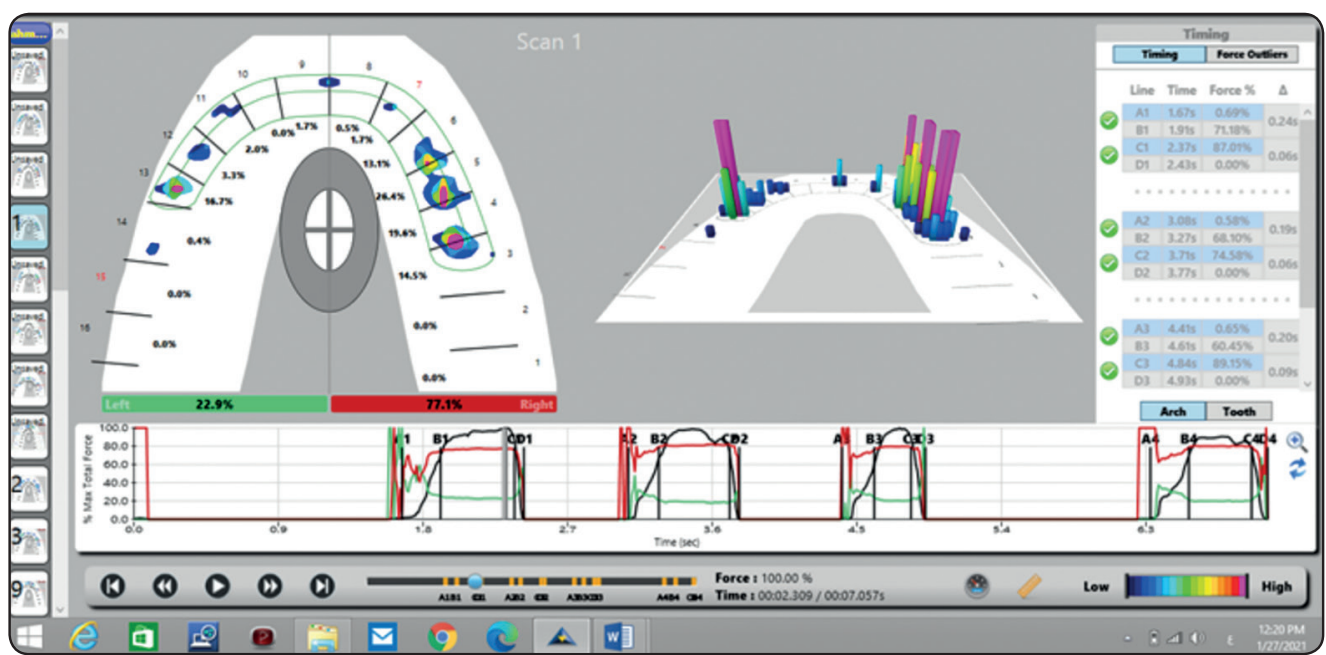

Fig. (5) 3D graph Zoom windows showing color-coded "traces" representing the forces applied on each teeth

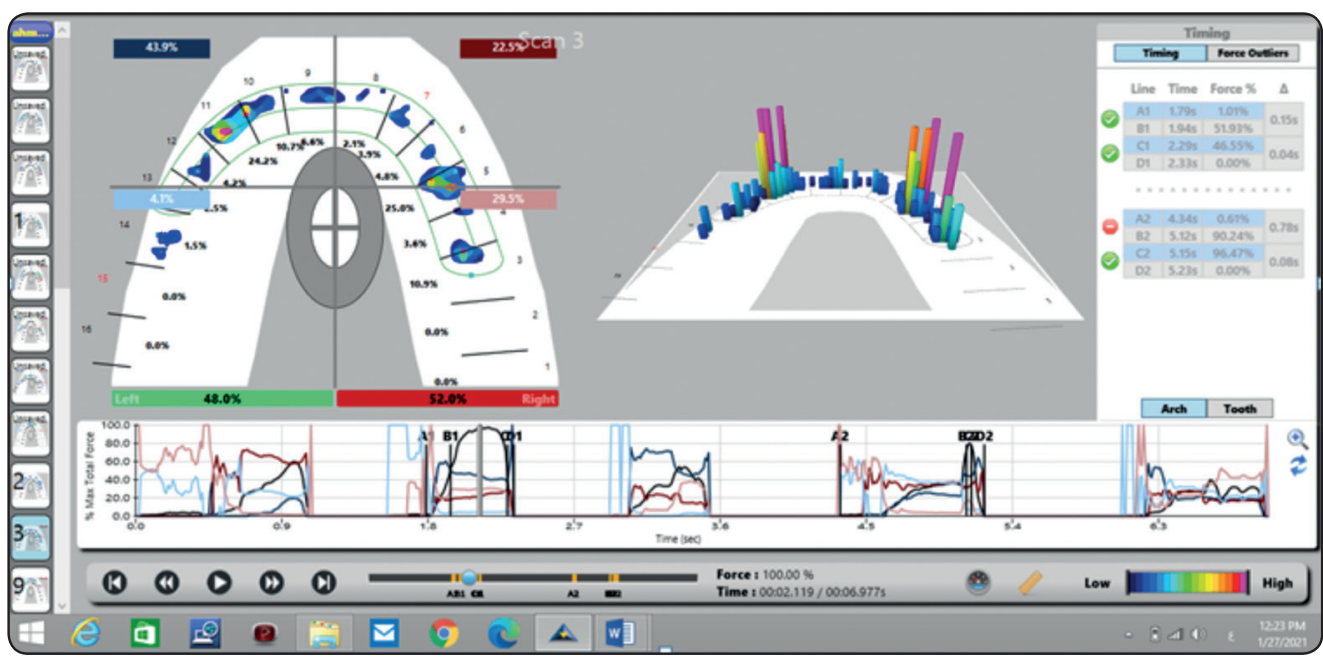

Fig. (6) 3D graph Zoom windows after occlusal adjustment and uniform force distribution. 
The same process were done to adjust contact at protrusive and lateral eccentric.

Patients were frequently recalled every 6 months for inspection, post hygiene maintenance and for CBCT radiographs at the time of implant loading, six, twelve and eighteen months postoperatively.

Marginal bone height change around the implants was evaluated using the measurement software system supplied with the CBCT radiographs. The bone height was measured by measuring the distance from the alveolar crest to the implant apex. The results were collected, tabulated and statistically analysed.

\section{RESULTS}

Data were presented as mean difference and standard deviation.Statistical analysis was performed with IBM ${ }^{\circledR}$ SPSS ${ }^{\circledR}$ (SPSS Inc., IBM Corporation, NY, USA) Statistics Version 25 for Windows. Testing the significance between the two studied groups for each surface was done by independent t-test. A probability value of $(\mathrm{P} \leq 0.05)$ was considered statistically significant.

Crestal bone height change in group I and group II during follow up period.

Six months after loading the mean value amount of crestal bone height change was 0.232 and 0.247 for group I and group II respectively.

12 months after loading the mean value amount of crestal bone height change was 0.212 and 0.278 for group I and group II respectively.

18 months after loading the mean value amount of crestal bone height change was 1 and 1.12 for group I and group II respectively.

As shown in table 1, Upon comparing the two groups, there was statistically insignificant difference between group I and II during the first 6 months, however there was a statically significant difference between the two group after 12 months and 18 months

TABLE (1): The mean and standard deviation values of crestal bone height change around the implants for Group I and Group II during follow up periods.

\begin{tabular}{|c|c|c|c|c|c|}
\hline \multirow{2}{*}{ Period } & \multicolumn{2}{|c|}{ Group I } & \multicolumn{2}{c|}{ Group II } & \multirow{2}{*}{ P-value } \\
\cline { 2 - 5 } & Mean & S.D & Mean & S.D & \\
\hline After 6 months & .232 & .024 & .247 & .049 & .681 \\
\hline After 12 months & .212 & .04 & .278 & .013 & $.019 * *$ \\
\hline After 18 months & 1 & .071 & 1.12 & .051 & $.028 * *$ \\
\hline
\end{tabular}




\section{DISCUSSION}

Prosthetic rehabilitation of edentulous mandible opposed by natural mandibular teeth is one of the most complex problems in removable prostheses. All attempts aimed at reducing the stresses induced by the opposing natural teeth to save the dental implants and the lower residual ridge.

Occlusal overloading and premature contacts being the proven factor for implants complications and failure. ${ }^{(14)}$ Diagnostic screening and Occlusal trauma is a major cause of occlusal derived pathology. ${ }^{(6)}$

Results of this study showed that crestal bone height loss was more in group II rather than group I during the follow up period and it showed significant difference during 12 and 18 months.

As $T$ scan is capable of quantifying relative forces with accurate information about timing of forces while articulating paper was the most common treatment medium used for occlusion, but force measurements, time measurement are the shortcomings faced in routine practice. T scan overcomes such shortcomings efficiently with adequate reliability and sensitivity. ${ }^{(15)}$

Articulating papers were also been used previously for determination of the location of contact for occlusal contacts but there was a problem with reliability of this procedure and modality in addition that it can also be deformed due to presence of saliva. ${ }^{(8)}$

The $t$ scan has higher sensitivity to the load applied, and reproducibility thus allow for better occlusal analysis and thus better occlusal adjustment ${ }^{(12)}$, also it has the ability to record, relative force intensity and timing thus allowing to evenly distribute the load allover the prosthesis and the dental implants ${ }^{(6)}$

Results of this study support the opinion of Majithia et al that T Scan III was is a reliable device to detect early contacts and could be effectively used to check occlusal balances. Also It could be used in full arch rehabilitation and other occlusal rehabilitation cases. The main advantage of a $\mathrm{T}$ Scan III analysis is that it could detect the amount of force as well as location of the highest intensity contacts of a single tooth which is very specific. ${ }^{(16)}$

It displays relative occlusal force information, so the clinician can minimize repeated errors of incorrect occlusal contact selection that often occur from relying just on the combination of dental articulating paper and patient feel. ${ }^{(17)}$

Articulating paper markings can be contaminated by the saliva and hence can cause misinterpretation of readings whereas in T Scan III the sensors are synthetic and resistant to salivary wetting of the sensors thus maintaining the accuracy of the recordings. ${ }^{(16)}$ The characteristic articulating paper marking is observed as a central area that is devoid of the colorant and surrounded by a peripheral rim of the dye. The density of these markings does not denote the force of the contact; instead, heavier contact tends to spread the mark peripheral to the actual location of the occlusal contact. ${ }^{(7)}$ Their thickness of $40 \mu$ is well above the thickness perception level of the patient and their relatively inflexible base material leads to the formation of a large number of pseudocontact markings. ${ }^{(7)}$

The T-Scan III determines the contact time sequencing and the percentage of relative occlusal force between numerous occlusal contacts and then displays them for all dynamic analysis. ${ }^{(18)}$ This enables the clinician to better identify many interfering contacts that are not readily identified by articulation paper markings. These occlusal corrective adjustments are made by selectively grinding the marks to obtain occlusal stability, multiple contacts throughout the arches that exhibit simultaneity and reduce stress on the occlusal contacts ${ }^{(19)}$ so it allows for better distribution of the forces along the supporting implants therefore the dental implants will be subjected to less loading resulting in reducing the bone loss around the implants. 


\section{CONCLUSION}

Within the limitation of this study, it could be concluded that the use of $t$ scan III device allow for better occlussal adjustment in case of implant supported lower single denture than articulating paper and resulting in less vertical bone loss around the supporting dental implants

\section{REFERENCES}

1. Hobkirk JA, Abdel-Latif HH, Howlett J, Welfare R, Moles DR. Prosthetic treatment time and satisfaction of edentulous patients treated with conventional or implant-stabilized complete mandibular dentures: a case-control study (part 2). Int J Prosthodont;22:13-9,2009.

2. Meijer HJA, Kuiper JH, Starmans FJM and Bosman FJM. : Stress distribution around dental implants: influence of superstructure, length of implant and height of mandible. J Prosthet Dent. 1992;68:96-102.

3. Miyata t. et al. "The influence of controlled occlusal overload on peri-implant tissue. Part 3 : A histological study in monkeys" The international Journal of Oral and Maxillofacial Implants 15 (2000): 425-431.

4. Schwarz M.S "Mechanical complications of dental implant”. Clinical oral implants Research 11(2000):156-158.

5. Kim Y et al. "Occlusal considerations in implant therapy : clinical guidelines with biomechanical rationale". Clinical Oral Implants Research 16(2005):26-35.

6. Pyakurel U, Long H, Jian F, Sun J, Zhu Y, Jha H, Lai W. Mechanism, accuracy and application of T-Scan system in dentistry-a review. J Nepal Dent Assoc 2013;13:52-6.

7. Carossa S, Lojacono A, Schierano G, Pera P. Evaluation of occlusal contacts in the dental laboratory: Infl uence of strip thickness and operator experience. Int J Prosthodont 2000;13:201-4.

8. Sidana V, Pasricha N, Makkar M, Banwait S. Computerized occlusal analysis: Review. Indian J Dent Sci. 2013;5:141-4.
9. Lyons MF, Sharkey SW, Lamey PJ. An evaluation of the TScan computerised occlusal analysis system. Int J Prosthodont 1992;5:166-72.

10. Ramakrishna Rajan Babu, Sanjna Vibhu Nayar. Occlusion indicators: A review .The Journal of Indian Prosthodontic Society $2007 ; 7: 170-174$.

11. Cerna M, Ferreira R, Zaror C, Navarro P, Sandoval P Validity and reliability of the T-Scan III for measuring force under laboratory conditions. J Oral Rehabil 2015; 42:544-51.

12. Koos B, Godt A, Schille C. et al. Precision of an Instrumentation-based Method of Analyzing Occlusion and its Resulting Distribution of Forces in the Dental Arch. J Orofac Orthop 2010;71,403-10

13. Trpevska V, Kovacevska G, Benedeti A, Jordanov B. T-SCAN III system diagnostic tool for digital occlusal analysis in orthodontics- a modern approach. J Med Sci 2014;2:155-60.

14. Dario LJ. How occlusal forces change in implants patients: a clinical research report. J Am Dent Assoc 1995;126: 1130-33.

15. Makofsky, H.W., Sexton, T.R., Diamond, D.Z., Sexton, M.T.,. The effect of head posture on muscle contact position using the T-scan system of occlusal analysis. Cranio ${ }^{\circledR}$ 1991;9:316-21.

16. Majithia I., Arora V., Kumar A., Saxena V. and Mittal M.: Comparison of articulating paper markings and T Scan III recordings to evaluate occlusal force in normal and rehabilitated maxillofacial trauma patients. Med. J. A. F. India. xxx; 1, 2014.

17. Kerstein R.B., Lowe M., Harty M., Radke J. A force reproduction analysis of two recording sensors of a computerized occlusal analysis system. Cranio. 2006;24:15-24

18. Maness W.L., Benjamin M., Podoloff R. Computerized occlusal analysis: a new technology. Quintessence Int. 1987;18(4):287-292.

19. Dawson P.E. vol. 1. Mosby and Co; St Louis (MO): 2007. p. 347. (Functional Occlusion: From TMJ to Smile Design). 